\title{
Correction to: Theoretical Considerations on Pedagogic Practice and Its Transformative Potential
}

\section{Correction to:}

Chapter 2 in: S. Leder, Transformative Pedagogic Practice, Education for Sustainability, https://doi.org/10.1007/978-981-13-2369-0_2

In the original version of this paper, image " 2.3 " appeared twice instead of image " 2.4 ". The original chapter has been updated with the change. 